Ophthalmologe $2021 \cdot 118: 287$

https://doi.org/10.1007/s00347-020-01205-3

Online publiziert: 18 . August 2020

(c) Springer Medizin Verlag $\mathrm{GmbH}$, ein Teil von Springer Nature 2020

Markus Gruber - Thomas Reinhard • Philip Maier

Klinik für Augenheilkunde, Universitätsklinikum Freiburg, Medizinische Fakultät, Albert-Ludwigs

Universität Freiburg, Freiburg, Deutschland

\title{
Verletzungen der Orbita durch Augenprothesen beim Tauchen
}

\section{Erwiderung}

Zum Leserbrief von Rokohl AC, Trester M, Hinkelbein J, Heindl LM (2020) Implosion von Augenprothesen - Bedeutung von Form und Material. Ophthalmologe. https://doi.org/10. 1007/s00347-020-01204-4

\section{Originalbeitrag \\ Gruber M, Reinhard T, Maier P (2020). Orbitale Blutung während eines Tauchgangs. Ophthal- mologe. https://doi.org/10.1007/s00347-020- 01144-z}

Wir danken den Kollegen Rokohl et al. sehr für den Leserbrief zu unserem Beitrag „Orbitale Blutung während eines Tauchunfalls“. Wie die Autoren völlig korrekt schildern, kommt es nur äußerst selten zu Problemen durch Augenprothesen. So sehen wir auch an der Klinik für Augenheilkunde des Universitätsklinikums Freiburg fast nie ernsthafte Komplikationen, was die Bedeutung des hier diskutierten Falls unterstreicht. Der in unserem Fallbericht erwähnte Patient trägt bereits seit 50 Jahren Augenprothesen aus Kryolithglas, nachdem er im 5. Lebensjahr sein Auge durch eine Pfeilverletzung verloren hatte. Die doppelwandige Augenprothese wird dabei jährlich durch einen Okularisten neu hergestellt, um Beeinträchtigungen der Augenprothese durch zu langes Tragen zu vermeiden. Seit vielen Jahren geht der Patient begeistert seinem Hobby, dem Tauchsport, mit Tauchtiefen über $40 \mathrm{~m}$ nach, wobei das Tragen seiner Augenprothese stets völlig problemlos war.

Weshalb es dann bei dem betreffenden Tauchgang offensichtlich zu einer Implo- sion der Prothese gekommen ist, bleibt unklar. Die möglichen Ursachen wie eine dünne Rückwand der Glasprothese oder starke Temperaturschwankungen werden in unserem Bericht sowie von den Kollegen Rokohl et al. in ihrem Leserbrief entsprechend diskutiert. Um in Zukunft erneute Probleme mit seiner Augenprothese zu vermeiden, verwendet der Patient beim Tauchen inzwischen eine einwandige Prothese aus Kryolithglas.

\section{Korrespondenzadresse}

\section{Dr. med. Markus Gruber}

Klinik für Augenheilkunde, Universitätsklinikum Freiburg, Medizinische Fakultät, Albert-Ludwigs Universität Freiburg

Killianstr. 5, 79106 Freiburg, Deutschland markus.gruber@uniklinik-freiburg.de

Interessenkonflikt. M. Gruber, T. Reinhard und P. Maier geben an, dass kein Interessenkonflikt besteht.

Für diesen Beitrag wurden von den Autoren keine Studien an Menschen oder Tieren durchgeführt. Für die aufgeführten Studien gelten die jeweils dort angegebenen ethischen Richtlinien. 